\title{
BMJ Open Quality Impact of full personal protective equipment on alertness of healthcare workers: a prospective study
}

\author{
Helena Jane Wells (i) , Meera Raithatha, Sabba Elhag, James Turner, Pranav Osuri, \\ Santhana Kannan
}

To cite: Wells HJ, Raithatha M, Elhag S, et al. Impact of full personal protective equipment on alertness of healthcare workers: a prospective study. BMJ Open Quality 2022;11:e001551. doi:10.1136/ bmjoq-2021-001551

Received 16 May 2021 Accepted 31 December 2021

\section{Check for updates}

(C) Author(s) (or their employer(s)) 2022. Re-use permitted under CC BY-NC. No commercial re-use. See rights and permissions. Published by BMJ.

Critical Care, Sandwell and West Birmingham Hospitals NHS Trust, Birmingham, UK

Correspondence to Dr Helena Jane Wells; helena.wells1@nhs.net

\section{ABSTRACT}

The COVID-19 pandemic has resulted in healthcare workers (HCWs) having to wear full personal protective equipment (FPPE) even for routine patient care. When worn for prolonged periods, FPPE has the potential to adversely affect the ability of HCW to carry out their tasks. This study aimed to assess the impact of FPPE on the alertness levels of HCWs in the intensive care unit.

Fifty HCWs were tested using psychomotor vigilance test (PVT) and serial sevens test (SST) on two occasions during their shift-once without FPPE and once after they had been in FPPE for at least 2 hours. The median time in FPPE was 2.5 hours. FPPE induced a significant increase in the average reaction time and the number of false responses in PVT. Subjects took longer to complete SST in FPPE compared with without it.

This study demonstrates that being in FPPE for as little as 2.5 hours can adversely affect HCW alertness. The findings of this study are relevant in terms of workforce environment considerations as the use of FPPE in certain settings is likely to become a regular feature.

\section{INTRODUCTION}

The COVID-19 pandemic brought many changes to practices of healthcare workers (HCWs) including the need to wear appropriate personal protective equipment (PPE) in potentially hazardous scenarios. The PPE can be cumbersome and uncomfortable for the individual. The condition of critically ill patients in the intensive care unit (ICU) can change acutely, requiring prompt and appropriate response from HCW. Therefore, it is desirable to minimise factors which can hinder this response. We hypothesised that the wearing of PPE may impact on the HCW ability to respond in a timely and efficient manner.

Even before the COVID-19 pandemic, HCWs in ICU wore PPE in their day-to-day work environment. This typically included the use of aprons and gloves for routine patient care. For specialist interventions and those requiring sterile precautions, additional measures such as gowns, face mask and a visor were required. The COVID-19 pandemic resulted in HCW having to wear full PPE (FPPE) even for routine patient care. FPPE consisted of a respirator mask (N95, FFP2 or FFP3), a body covering suit, visor, gloves and hat (figure 1). When worn for prolonged periods, FPPE has the potential to adversely affect the ability of HCW to carry out their tasks. This study aimed to assess the impact of FPPE on the alertness levels of HCW in ICU.

Research within the healthcare sector in relation to the impact of PPE on functional ability has been limited. In a survey, over half of surgeons felt that their surgical performance had been hampered by the wearing of PPE. ${ }^{1} \mathrm{HCW}$ have reduced dexterity and impaired visibility when wearing PPE. ${ }^{2}$ PPE increased the time taken to draw up medication and resulted in less amount being drawn up. ${ }^{3}$ It is to be noted that PPE used in this study was designed for chemical, biological, radiation or nuclear incidents and is more complex than that used by HCW in hospitals. Most HCWs in ICU need to work in shift patterns which can result in sleep deprivation. This in turn has potential to adversely affect several aspects of cerebral cortical function and reduced alertness. ${ }^{4}$ This study intended to assess the impact of wearing FPPE on HCW alertness level and cognition, while working in the ICU.

\section{METHODS}

Study design and setting

This was a single-centre prospective study on staff working in the two ICU across sites with a total of 30 beds. HCW were invited to participate voluntarily via email. This included doctors, nurses, physiotherapists, healthcare assistants and redeployed staff from other areas of the hospital. Staff were then approached during their working shift by a delegated member of the research team for consent. Inclusion criteria consisted of all HCW staff who agreed to take part 


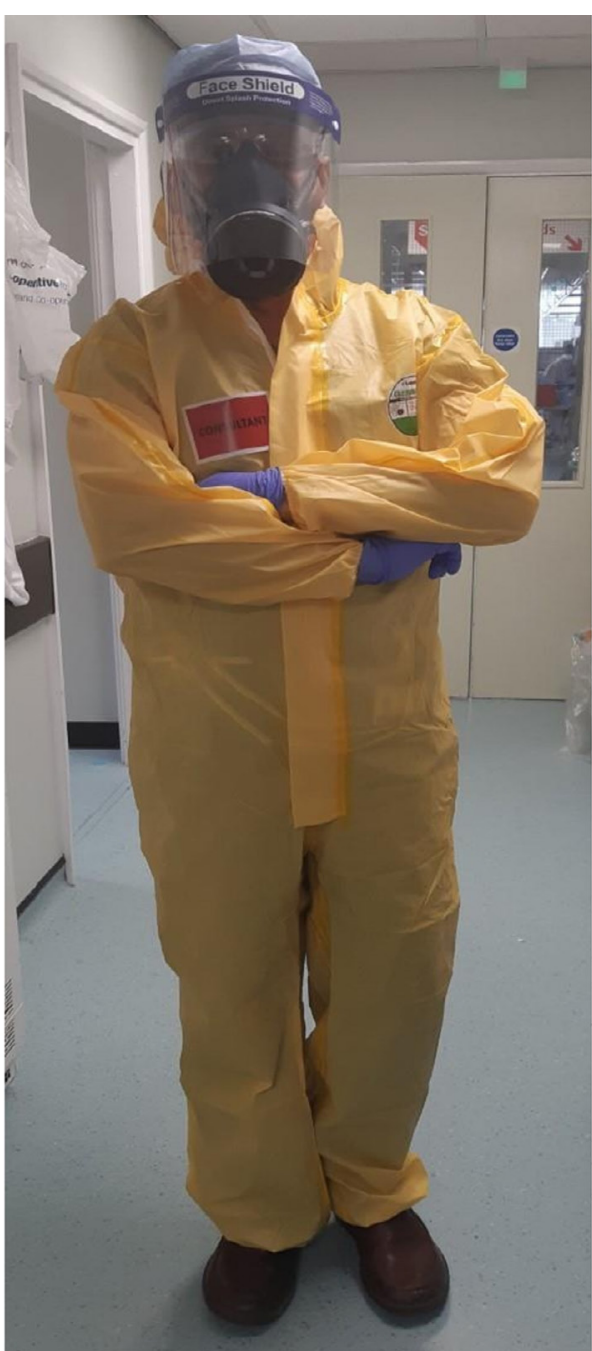

Figure 1 Full personal protective equipment used by healthcare workers.

voluntarily without any incentive. Exclusion criteria included any staff members not required to wear PPE as part of their job role, staff wearing any other type of FPPE such as powered air-purifying respirator, refusal of consent, withdrawal midway through the study due to any reason including health, claustrophobia or subject wish, self-reported significant visual impairment, and less than 6 hours of sleep in the previous 24 hours, including less than 4 hours of continuous sleep. Subjects were only included if their participation did not affect patient care and another HCW was available to provide cover. The aim was to recruit at least 50 subjects for the study. Subjects were recruited consecutively as per their availability. The research team members were excluded from participation.

\section{Patient and public involvement statement}

There was no patient involvement in this study.

\section{Testing methods}

A set of two psychomotor tests was used to assess response times and error rates. Testing was performed by research team members who were trained in the administration of the tests and data recording.

Subjects were assessed either during their day or night shift. Each subject was administered the set of tests twice and hence acted as their own control. One set was conducted with the subject not wearing any PPE (control arm) and another when they were still in FPPE after they had been in it for at least 2 hours (FPPE arm). To minimise the chance of subjects practising the tests at home, both sets were completed in the same shift with no trial sessions. The test without FPPE was done prior to the start of the shift or during a rest break in the middle of shift. If the subject was tested in the FPPE arm first, a time interval of at least 1 hour was allowed for the subject to be out of FPPE before administering the tests for the control arm. Testing was performed in a quiet area with no distractions. For the FPPE arm, a vacant isolated room within the ICU was used for testing where both the subject and the research team member were in FPPE. Subjects were asked not to disclose the nature of tests to other colleagues.

The two testing tools used were the psychomotor vigilance test (PVT) and the serial sevens test (SST). PVT is a chronometric measure of an individual's reaction to specified small changes in a labile environment. ${ }^{5}$ Subjects are instructed to respond to a digital signal on a computer screen by pressing a key. It was performed via the 'PVT Research Tool' App, on a portable electronic device. This recorded the subject's reaction times when a red dot appeared on the screen and was conducted for a duration of $5 \mathrm{~min}$. The average, minimum and maximum response time and number of false responses were recorded. For the SST, participants were asked to subtract 7 from 100 in a serial fashion. ${ }^{6}$ For each subtraction error made, participants were promptly provided with the correct answer and instructed to continue the subtraction procedure beginning with the corrected response. The time to completion and the number of errors was recorded. The order of tests was kept the same whereby PVT was followed by SST for all subjects.

For statistical analysis, categorical variables are described as numbers and proportions. Continuous variables are described as mean and SD or median and IQR. Changes in psychometric test scores with and without FPPE were analysed using Wilcoxon signed ranks test. Difference in test scores between day and night shift was analysed using Mann-Whitney test.

\section{RESULTS}

Sixty-two eligible HCW were approached to participate. Fifty subjects were included in the final analysis (figure 2). The study was done over a period of 9 months from July 2020. The study took longer than anticipated due to an interim period when the number of COVID-19 patients in ICU fell and there were less designated bays which required FPPE.

The demographics of subjects is shown in table 1 . Twenty-eight subjects were from morning shift, 12 during 


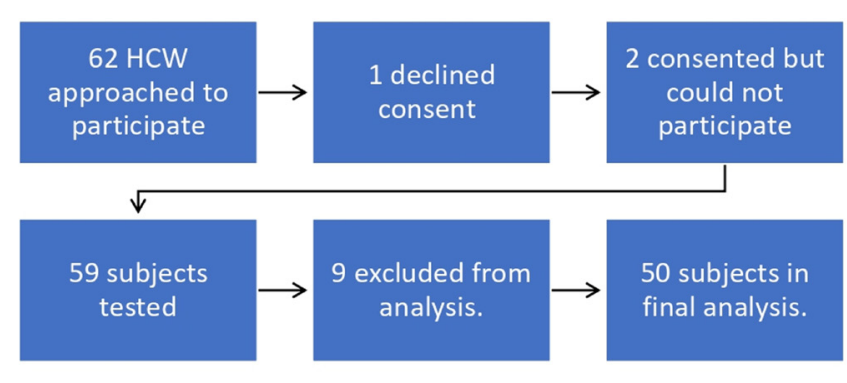

Exclusion reasons -

3 did not complete both sets of tests.

6 protocol violations ( 2 recruited in spite of inadequate sleep, 2 did not have enough interval between tests, 2 did not spend sufficient time in full personal protective equipment).

Figure 2 CONSORT flow diagram.

the afternoon shift and 10 from night shift. Two-thirds of the subjects were under the age of 40 years. Medical staff comprised $62 \%$ of the total including four consultants. The mean (SD) sleep duration in the 24 hours period prior to testing was $6.99(0.93)$ hours. The median time in FPPE prior to testing was 2.5 hours with a maximum time of 4 hours. Thirty-three subjects $(66 \%)$ tested were in FPPE for the first set of tests.

The effect of FPPE on the test scores is shown in table 2. FPPE induced a significant increase in the average and minimum reaction time along with the number of false responses in PVT. Subjects also took longer to complete SST and had higher number of errors with FPPE compared with without it. There was a statistically significant difference in the PVT average reaction time scores with FPPE between those tested in day vs night shift $(\mathrm{p}<0.05)$.

\section{DISCUSSION}

This study demonstrates a significant reduction in alertness levels of HCW when wearing FPPE. There have been no similar studies in the literature before.

The condition of patients in ICU can deteriorate significantly in a short space of time. Hence, prompt and accurate response from HCW is essential to manage these changes. This in turn requires a high level of alertness

\begin{tabular}{lc}
\hline Table 1 & Demographics of the study subjects \\
\hline Characteristic & $\mathbf{n}(\%)$ \\
\hline Male & $23(46)$ \\
Female & $27(54)$ \\
Age range & $16(32)$ \\
$21-30$ years & $18(36)$ \\
$31-40$ years & $11(22)$ \\
$41-50$ years & $5(10)$ \\
$51-60$ years & $31(62)$ \\
Job title-doctor & $19(38)$ \\
\hline Job title-nurse &
\end{tabular}

where the information from monitors and ventilators is processed rapidly and an appropriate response is formulated. Factors which can impair alertness can lead to either a delayed, inadequate or incorrect response.

PPE can adversely impact the functioning of HCW in a variety of ways ranging from inducing discomfort to errors in motor tasks. Less than a quarter of workers in a metal refinery plant rated their PPE comfort level as acceptable. ${ }^{7}$ HCWs with pre-existing headache disorders, or those wearing PPE for prolonged periods, were more likely to report new headaches or exacerbation of their underlying condition. ${ }^{8}$ Carbon dioxide retention could also be a contributory factor as it has been shown to rise by $2 \%$ when speaking or undertaking sedentary work in a respirator mask. ${ }^{9}$ Another study showed that the full-face negative pressure respirator mask increased the error rate by subjects during tests for cognitive performance without affecting the task completion time. ${ }^{10}$

Psychomotor tests have been used as a surrogate marker of complex cerebral functions such as alertness and information processing. ${ }^{511}$ PVT and SST were chosen as they have been used in psychological assessments. They are easy to understand, execute and perform. Errors during PVT are believed to represent perceptual, processing or executive failures in the central nervous system. Impairment in executive functioning is reflected in another measure, the PVT count of false responses (responding when no stimulus is presented). PVT has been shown to be devoid of practice or learning effects. The presence of the app allows accurate recording of results. They only took a few minutes to perform making it not too arduous for the subjects. SST has been used in neuropsychological tests as putative measures of complex attention and speed of information processing. ${ }^{12}$ As the subjects acted as their own control, their intellectual ability was not a factor.

FPPE adversely affected the performance in both PVT and SST indicating that multiple components of functioning of the cerebral functions are adversely impacted. Despite only 10 subjects being tested during their night shift, there was a significant difference in the PVT average reaction time with FPPE when compared with daytime staff. FPPE appears to add to the mental strain of working during a night shift.

This study provides evidence that being in FPPE for as little as 2.5 hours can adversely affect HCW alertness. Currently, some HCW spend around 4 hours in FPPE before their break. In the peak of the pandemic, nurse to patient ratio was increased from 1: 1 to $1: 3$ to accommodate the higher patient load. This along with working in FPPE had potential to increase mental fatigue among HCW. The findings of this study are relevant in terms of workforce environment considerations as the use of FPPE in certain settings is likely to become a regular feature. In situations which demand working in FPPE, certain mitigating factors could be considered to counter the risk: allocation of breaks in a manner which allows 'fresh' staff to be mixed with those in FPPE for a longer time, remote 


\begin{tabular}{|c|c|c|c|c|c|}
\hline Test & Variable & Without FPPE & With FPPE & Test statistic & $P$ value \\
\hline PVT & Average reaction time & $0.5229 \mathrm{~s}$ & $0.5742 \mathrm{~s}$ & 165 & $<0.001$ \\
\hline PVT & Maximum reaction time & $0.9875 \mathrm{~s}$ & $1.2211 \mathrm{~s}$ & 505 & $>0.05$ \\
\hline PVT & Average no of false responses & 0.22 & 0.36 & 227 & $<0.001$ \\
\hline SST & Average no of errors & 1.04 & 1.38 & 271 & $<0.001$ \\
\hline
\end{tabular}

PVT, psychomotor vigilance test; SST, serial sevens test.

monitoring from someone not in FPPE or reducing the number of 'consecutive night shifts', etc.

This study has certain limitations as it does not prove that the adverse effect of FPPE automatically translates to adverse outcomes. It is well known that in real life, there is a high potential for errors of omission, commission and near misses. ${ }^{13}$ Any adverse event is usually the culmination of a series of events progressing beyond a certain threshold. In practice, most of any errors are picked up well in time and acted on. It would be extremely challenging to prove that a particular event was a direct result of use of FPPE. On the other hand, if the potential for errors is reduced, it should lead to improvement in safety.

In the original protocol, the intention was to assess subjects after they had been in FPPE for at least 3 hours. However, due to practical constraints with the excessive patient load and the need to balance availability of staff with suitable skill mix, this proved difficult to achieve. Hence, a duration of 2 hours in FPPE was deemed acceptable for the purposes of this study.

The observed decline in alertness may have also been influenced by general fatigue accumulated during a shift between the two set of tests. A further 'control group' of subjects without FPPE, but undergoing the two set of tests with the same time interval as the study subjects, would have helped to highlight whether this was a significant contributor. Approximately two-thirds of subjects in this study had their first set of tests in FPPE. If 'work related fatigue' was a factor, the difference between the two sets of tests would have been a lesser magnitude. It would have been optimal if all the subjects were tested in the same order with regard to FPPE. Practical constraints meant that this was not always possible.

We excluded individuals with insufficient sleep from this study. In real life, some HCW are unable to get sufficient sleep prior to their shifts due to a multitude of factors. ${ }^{4}$ Hence, use of FPPE is likely to impair their alertness levels to a greater extent in those individuals. One other potential confounding factor was the influence of 'memory effect' on the results especially for SST. This was less likely as the test was only repeated once. Even if it was the case, the study still showed a significant difference between the test results with and without FPPE. The same applies to the possibility that some subjects were aware of the nature of tests from their colleagues.
In summary, being in FPPE adversely affected the alertness of HCW when tested using PVT and SST. This should be taken into consideration for staff working patterns in areas such as ICU where a high degree of vigilance is required.

Acknowledgements We thank the clinical research team at Sandwell and West Birmingham NHS Trust for their help in instigating the project.

Contributors HJW, SE, JT, PO and SK designed the study and developed the protocol and analysis plan. HJW, MR, SE, JT and PO collected and analysed the data. HJW, MR and SK drafted the manuscript. All authors contributed to the study design and revised the manuscript. HJW is responsible for the overall content as guarantor. The corresponding author attests that all listed authors meet authorship criteria and that no others meeting the criteria have been omitted.

Funding The authors have not declared a specific grant for this research from any funding agency in the public, commercial or not-for-profit sectors.

Competing interests None declared.

Patient and public involvement Patients and/or the public were not involved in the design, or conduct, or reporting, or dissemination plans of this research.

Patient consent for publication Not applicable.

Ethics approval Approval from relevant national bodies was obtained prior to the start of study (Health Research authority, England and Health \& Care Research, Wales. Integrated Research Application Service project ID: 286330 and Research and Ethics Committee reference: 20/HRA/3596).

Provenance and peer review Not commissioned; externally peer reviewed.

Data availability statement Data are available on reasonable request. Data relating to the study not already included in the article ARE available on reasonable request subject to approval of release of the same from the institution.

Open access This is an open access article distributed in accordance with the Creative Commons Attribution Non Commercial (CC BY-NC 4.0) license, which permits others to distribute, remix, adapt, build upon this work non-commercially, and license their derivative works on different terms, provided the original work is properly cited, appropriate credit is given, any changes made indicated, and the use is non-commercial. See: http://creativecommons.org/licenses/by-nc/4.0/.

ORCID iD

Helena Jane Wells http://orcid.org/0000-0001-8126-7097

\section{REFERENCES}

1 Yánez Benítez C, Güemes A, Aranda J, et al. Impact of personal protective equipment on surgical performance during the COVID-19 pandemic. World J Surg 2020;44:2842-7.

2 Loibner M, Hagauer S, Schwantzer G, et al. Limiting factors for wearing personal protective equipment (PPE) in a health care environment evaluated in a randomised study. PLOS One 2019;14:e0210775.

3 Castle N, Bowen J, Spencer N. Does wearing CBRN-PPE adversely affect the ability for clinicians to accurately, safely, and speedily draw up drugs? Clin Toxicol 2010;48:522-7. 
4 Ganesan S, Magee M, Stone JE, et al. The impact of shift work on sleep, alertness and performance in healthcare workers. Sci Rep 2019;9:4635.

5 Wilkinson RT, Houghton D. Field test of arousal: a portable reaction timer with data storage. Hum Factors 1982;24:487-93.

6 LaMarche JA, Alexander RW, Stanford LD. A descriptive study of cognitive efficiency: verbal and numerical cognitive fluency. Archives of Clinical Neuropsychology 1994;9:152.

7 Akbar-Khanzadeh F. Factors contributing to discomfort or dissatisfaction as a result of wearing personal protective equipment. J Hum Ergol 1998;27:70-5.

8 Ong JJY, Bharatendu C, Goh Y, et al. Headaches Associated With Personal Protective Equipment - A Cross-Sectional Study Among Frontline Healthcare Workers During COVID-19. Headache 2020;60:864-77.
9 Smith CL, Whitelaw JL, Davies B. Carbon dioxide rebreathing in respiratory protective devices: influence of speech and work rate in full-face masks. Ergonomics 2013;56:781-90.

10 AlGhamri AA, Murray SL, Samaranayake VA. The effects of wearing respirators on human fine motor, visual, and cognitive performance. Ergonomics 2013;56:791-802.

11 Loh S, Lamond N, Dorrian J, et al. The validity of psychomotor vigilance tasks of less than 10-minute duration. Behav Res Methods Instrum Comput 2004;36:339-46.

12 Williams MA, LaMarche JA, Alexander RW, et al. Serial 7S and alphabet backwards as brief measures of information processing speed. Archives of Clinical Neuropsychology 1996;11:651-9.

13 Rothschild JM, Landrigan CP, Cronin JW, et al. The critical care safety study: the incidence and nature of adverse events and serious medical errors in intensive care. Crit Care Med 2005;33:1694-700. 